\title{
Analytic solution to $N$ vs. $M$ photon phase control in an open two-level system
}

\author{
Heekyung Han and Paul Brumer \\ Chemical Physics Theory Group, \\ Department of Chemistry, and \\ Center for Quantum Information and Quantum Control \\ University of Toronto \\ Toronto, Canada M5S $3 \mathrm{H} 6$
}

(Dated: November 16, 2018)

\begin{abstract}
Decoherence effects on the traditional $N$ vs. $M$ photon coherent control of a two-level system are investigated, with 1 vs. 3 used as a specific example. The problem reduces to that of a two-level system interacting with a single mode field, but with an effective Rabi frequency that depends upon the fundamental and third harmonic fields. The resultant analytic control solution is explored for a variety of parameters, with emphasis on the dependence of control on the relative phase of the lasers. The generalization to off-resonant cases is noted.
\end{abstract}




\section{INTRODUCTION}

Coherent control of atomic and molecular dynamics using optical fields has attracted much attention, both theoretically and experimentally [1, 2, 3]. Thus far, most theoretical work has focused on the idealized case of isolated systems, where loss of quantum phase information due to decoherence, i.e. coupling to the environment, is ignored. Such effects are, however, crucial to control in realistic systems, since loss of phase information results in loss of control. For this reason efforts to understand control in external environments 4] 7] and to compensate for the resultant decoherence (e.g., 8]-[15]) are of great interest.

There exist a number of basic interference schemes [1] that embody the essence of coherent control. One is the $N$ vs. $M$ photon scenario where control results from interference between state excitation using $N$ and $M$ photons simultaneously. In this letter we provide an analytic solution for control in the two-level $N$ vs. $M$ photon control scenario in the presence of decoherence. For simplicity, we examine the 1 vs. 3 photon case, although the solutions obtained below apply equally well to the $N$ vs. $M$ photon case, with obvious changes in the input Rabi frequencies and relative laser phases.

In 1 vs. 3 photon control [16] a continuous wave electromagnetic field composed of a superposition of a fundamental and third harmonic wave is incident on a system. By varying the relative phase and amplitude of the fundamental and the third harmonic one can alter the population of the state excited by the incident field. Clearly, decoherence can be expected to diminish the 1 vs. 3 photon induced interference, and hence the control over excitation. Although extensive theoretical [16] - 20] and experimental [21] - 25] studies have been carried out on the 1 vs. 3 photon coherent control scenario, there has been no serious examination of the stability of this control scheme in an external environment, barring a derivation of a simple analytical expression for the autoionization of a two-level atomic system for weak laser intensities, using the rate approximation [18]. Amongst the various possible influences of an environment on a system we focus on the loss of phase coherence, that is, dephasing. Dephasing is expected to occur on a time scale more relevant to control, since the duration of control field can be on the order of a picosecond or less, wheras the typical time scale for energy transfer is considerably longer [26, 27].

In this paper we show that the 1 vs. 3 photon phase control scenario (which controls the population) in a two-level system, when coupled to an environment, reduces to the 
analytically soluble monochromatic field case, but with an effective Rabi frequency that is determined by the relative phase and amplitudes of the two fields. Sample results for control as a function of relative laser phase in the presence of dephasing are then provided. The possiblity of solving the off-resonance case is also noted.

\section{II. $1+3$ PHOTON CONTROL}

\section{A. Formalism}

Consider a two-level bound system interacting with an continuous wave (CW) electromagnetic field and assume that the energy levels undergo random Stark shifts without a change of state during collisions with an external bath, e.g., elastic collisions between atoms in a gas. The CW field $E(t)$ is treated classically, and the ground and the excited energy eigenstates states, of energy $E_{1}$ and $E_{2}$ are denoted $|1\rangle$ and $|2\rangle$, respectively.

In general, the system density operator $\rho$ obeys the Liouville equation,

$$
\frac{\partial \rho}{\partial t}=-\frac{i}{\hbar}[\hat{H}(t), \rho]-\mathcal{R} \rho
$$

Here $\hat{H}(t)=\hat{H}_{\text {atom }}+\hat{H}_{\text {int }}$, where the free atomic Hamiltonian term is

$$
\hat{H}_{\text {atom }}=E_{1}|1\rangle\left\langle 1\left|+E_{2}\right| 2\right\rangle\langle 2|
$$

and the atom-field interaction term within the dipole approximation is

$$
\hat{H}_{\text {int }}=-E(t)[\langle 1|d| 2\rangle|1\rangle\langle 2|+\langle 2|d| 1\rangle| 2\rangle\langle 1|]
$$

with electric dipole operator $d$. The second term in Eq. (11), $\mathcal{R}$, is a dissipative term that can have a variety of nonequivalent forms associated with various master equations. Below we assume simple exponential dephasing of the off-diagonal $\rho_{i j}$.

In the simplest 1 vs. 3 control scenario, a two-level system is subject to the linearly polarized laser field:

$$
E(t)=\frac{1}{2}\left[\mathcal{E}_{f} e^{i \omega_{f} t} e^{-i \phi_{f}}+\mathcal{E}_{h} e^{i \omega_{h} t} e^{-i \phi_{h}}+\text { c.c. }\right]
$$

where $\mathcal{E}_{j}$ is the real time-independent amplitude and $\phi_{j}$ is the phase of the corresponding field, with $j=h, f$. Here the subscripts $f, h$ denotes the fundamental and its third harmonic, 
and "c.c." denotes the complex conjugate of the terms that precede it. The fields have frequencies $\omega_{f}$ and $\omega_{h}=3 \omega_{f}$, chosen so that the third-harmonic and the three fundamental photons are on resonance with the transition from the ground state $|1\rangle$ to the excited state $|2\rangle$. In the standard scenario [1, 2, 16], control is obtained by changing the relative phase and amplitudes of two fields, which results in the alteration of the degree of interference between the two pathways to the excited state.

Within the rotating-wave approximation, the slowly varying density-matrix elements of the states $|1\rangle$ and $|2\rangle, \sigma_{i i}=\rho_{i i},(i=1,2)$ and $\sigma_{21}=\rho_{21} e^{3 i\left(\omega_{f} t+\phi_{f}\right)}$ obey the following set of equations:

$$
\begin{aligned}
\frac{\partial \sigma_{11}}{\partial t} & =-\operatorname{Im}\left[\left(\mu_{12}^{(3)} \mathcal{E}_{f}^{3} / \hbar+\mu_{12} \mathcal{E}_{h} e^{i \phi} / \hbar\right) \sigma_{21}\right] \\
\frac{\partial \sigma_{22}}{\partial t} & =\operatorname{Im}\left[\left(\mu_{12}^{(3)} \mathcal{E}_{f}^{3} / \hbar+\mu_{12} \mathcal{E}_{h} e^{i \phi} / \hbar\right) \sigma_{21}\right] \\
\frac{\partial \sigma_{21}}{\partial t} & =-\gamma_{p} \sigma_{21}+\frac{i}{2}\left(\mu_{21}^{(3)} \mathcal{E}_{f}^{3} / \hbar+\mu_{21} \mathcal{E}_{h} e^{-i \phi} / \hbar\right)\left(\sigma_{11}-\sigma_{22}\right),
\end{aligned}
$$

with

$$
\mu_{12}^{(3)} \equiv \frac{1}{(2 \hbar)^{2}} \sum_{n, m} \frac{\mu_{1 n} \mu_{n m} \mu_{m 2}}{\left(\omega_{n 1}-\omega_{f}\right)\left(\omega_{f}-\omega_{2 m}\right)}
$$

Here $\gamma_{p}$ is the dephasing rate, $\omega_{n m}$ is the frequency difference between levels $|n\rangle$ and $|m\rangle$ and $\mu_{n m} \equiv\langle n|d| m\rangle$. The quantities $\mu_{12}$ and $\mu_{12}^{(3)}$ denote the one-photon matrix element for the harmonic field and the effective three-photon matrix element for the fundamental field for the $|1\rangle \rightarrow|2\rangle$ transition. Below, we use $\mu \equiv \mu_{12}$ and $\mu^{(3)} \equiv \mu_{12}^{(3)}$, omitting the subscripts for simplicity. The controllable relative phase is $\phi=\phi_{h}-3 \phi_{f}$.

It is convenient to define the one- and three-photon Rabi frequencies by $\Omega_{h}=\mu \mathcal{E}_{h} / \hbar$ and $\Omega_{f}=\mu^{(3)} \mathcal{E}_{f}^{3} / \hbar$, given in terms of their amplitudes and phases, by $\Omega_{h}=\left|\Omega_{h}\right| e^{i \theta_{h}}$ and $\Omega_{f}=\left|\Omega_{f}\right| e^{i \theta_{f}}$. Note that, although $\mu$ and $\mu^{(3)}$ are real for a bound system, we derive all the equations under the assumption that they can be complex so that the analysis can be extended to complex matrix elements arising in transitions to the continuum. Since $\mathcal{E}_{h}$ and $\mathcal{E}_{f}$ are real and positive, $\theta_{h}$ and $\theta_{f}$ are determined by $\mu$ and $\mu^{(3)}$ :

$$
\begin{aligned}
e^{i \theta_{h}} & =\mu /|\mu|, \\
e^{i \theta_{f}} & =\mu^{(3)} /\left|\mu^{(3)}\right| .
\end{aligned}
$$

To amalgamate these Rabi frequencies and the relative laser phase of $\phi$, we define the 
effective Rabi frequency $\Omega_{\mathrm{eff}}$ :

$$
\Omega_{\mathrm{eff}} e^{i \theta} \equiv \Omega_{h} e^{i \phi}+\Omega_{f}=\left|\Omega_{h} e^{i \phi}+\Omega_{f}\right| e^{i \theta},
$$

where $\Omega_{\text {eff }}$ is real and positive. Here $\Omega_{\text {eff }}$ and $\theta$ are related to $\Omega_{h}$ and $\Omega_{f}$ as

$$
\begin{aligned}
\Omega_{\mathrm{eff}} & =\sqrt{\left|\Omega_{h}\right|^{2}+\left|\Omega_{f}\right|^{2}+2\left|\Omega_{h} \Omega_{f}\right| \cos \Phi} \\
\tan \theta & =\left[\sin \left(\phi+\theta_{h}\right)+\frac{\left|\Omega_{f}\right|}{\left|\Omega_{h}\right|} \sin \theta_{f}\right] /\left[\cos \left(\phi+\theta_{h}\right)+\frac{\left|\Omega_{f}\right|}{\left|\Omega_{h}\right|} \cos \theta_{f}\right],
\end{aligned}
$$

where

$$
\Phi=\phi+\theta_{h}-\theta_{f}
$$

It is worth noting some features of $\Omega_{\text {eff }}$ that are evident from Eq. (10). First, the total excitation probability obtained in lowest order perturbation theory for 1 vs. 3 photon phase control in a two-level system [16] is proportional to $\Omega_{\mathrm{eff}}$. Hence, $\Omega_{\mathrm{eff}}$ can be used to predict the controlled population, and its dependence on $\phi$, when the fields are weak. Further (see below), $\Omega_{\text {eff }}$ plays a major role in determining the transient behavior of the excited state population for any field intensity in the absence or presence of dephasing. Second, the interference term in Eq. (10) can be controlled by varying $\Phi$, that is, by manipulating the relative phase $\phi$ of the two fields. Since $\mu$ and $\mu^{(3)}$ are real in a bound system, possible values of $\theta_{h}-\theta_{f}$ are 0 and $\pm \pi$. When $\theta_{h}-\theta_{f}=0$, i.e. , $\mu \mu^{(3)}>0, \cos \Phi=\cos \phi$. On the other hand, when $\theta_{h}-\theta_{f}= \pm \pi$, i.e. , $\mu \mu^{(3)}<0, \cos \Phi=-\cos \phi$. Thus, opposite interference effects are observed depending on the signs of $\mu$ and $\mu^{(3)}$. Third, ||$\Omega_{h}|-| \Omega_{f}|| \leq \Omega_{\text {eff }} \leq\left|\Omega_{h}\right|+\left|\Omega_{f}\right|$ so that maximal interference effects occur when $\left|\Omega_{h}\right|=\left|\Omega_{f}\right|$. If $\left|\Omega_{h}\right| \neq\left|\Omega_{f}\right|$, the smallest $\Omega_{\text {eff }}$ is not zero, and thus complete destructive interference, that is, zero excitation from the ground to the excited state, does not occur.

Rewriting Eq. (15) in terms of $\Omega_{\mathrm{eff}}$ and $\theta$,

$$
\begin{aligned}
& \frac{\partial \sigma_{11}}{\partial t}=-\operatorname{Im}\left[\Omega_{\mathrm{eff}} e^{i \theta} \sigma_{21}\right] \\
& \frac{\partial \sigma_{22}}{\partial t}=\operatorname{Im}\left[\Omega_{\mathrm{eff}} e^{i \theta} \sigma_{21}\right] \\
& \frac{\partial \sigma_{21}}{\partial t}=-\gamma_{p} \sigma_{21}+\frac{i}{2}\left[\Omega_{\mathrm{eff}} e^{-i \theta}\right]\left(\sigma_{11}-\sigma_{22}\right) .
\end{aligned}
$$

and introducing $u=2 \operatorname{Re}\left(\sigma_{12} e^{-i \theta}\right), v=2 \operatorname{Im}\left(\sigma_{12} e^{-i \theta}\right)$, and $w=\sigma_{22}-\sigma_{11}$, gives

$$
d u / d t=-\gamma_{p} u
$$




$$
\begin{array}{r}
d v / d t=-\gamma_{p} v+\Omega_{\mathrm{eff}} w \\
d w / d t=-\Omega_{\mathrm{eff}} v
\end{array}
$$

The resultant equations are now of standard form 28], but with $\Omega_{\text {eff }}$ replacing the Rabi frequency of the single field case discussed in Ref [28]. Note that the longtime steadystate solution to Eqs. (16) to (18) is found by setting $d u / d t=d v / d t=d w / d t=0$, giving $u(t \rightarrow \infty)=v(t \rightarrow \infty)=w(t \rightarrow \infty)=0$. This implies that, regardless of initial conditions and for sufficiently large time, pure dephasing leads to an equilibrium state with equal populations in the ground and the excited states and with no remaining coherence.

Substituting Eq. (17) into Eq. (18) gives a simple equation for $w$ :

$$
d^{2} w / d t^{2}+\gamma_{p} d w / d t+\Omega_{\mathrm{eff}}^{2} w=0
$$

In the important case where initially the ground state is populated and the coherence is zero [i.e., $w(0)=-1, u(0)=v(0)=0$ ], the excited state population $\rho_{22}=\sigma_{22}$ is given by

$$
\begin{gathered}
\rho_{22}=-\frac{e^{-\frac{\gamma_{p} t}{2}}}{2}\left[\cos (s t)+\frac{\gamma_{p}}{2 s} \sin (s t)\right]+\frac{1}{2} \quad \text { for } \gamma_{\mathrm{p}}<2 \Omega_{\mathrm{eff}}, \\
\rho_{22}=\frac{\left[-\lambda_{2} e^{\lambda_{1} t}+\lambda_{1} e^{\lambda_{2} t}\right]}{2\left(\lambda_{2}-\lambda_{1}\right)}+\frac{1}{2} \quad \text { for } \gamma_{p}>2 \Omega_{\mathrm{eff}}, \\
\rho_{22}=-\frac{e^{-\frac{\gamma_{p} t}{2}}}{2}\left(1+\frac{\gamma_{p} t}{2}\right)+\frac{1}{2} \quad \text { for } \gamma_{p}=2 \Omega_{\mathrm{eff}}
\end{gathered}
$$

where $s=\frac{1}{2} \sqrt{4 \Omega_{\mathrm{eff}}^{2}-\gamma_{p}^{2}}$, and $\lambda_{1,2}=\frac{1}{2}\left[-\gamma_{p} \pm \sqrt{\gamma_{p}^{2}-4 \Omega_{\mathrm{eff}}^{2}}\right]$. The general behavior of the solution is seen to be determined by relative size of the dephasing time and the period of the Rabi oscillation. Analogous analytic results can be obtained for $\sigma_{12}$ which decays with rate $\gamma_{\mathrm{p}}$. If the external field is intense enough so that $\gamma_{p}<2 \Omega_{\mathrm{eff}}$, then $\rho_{22}$ shows oscillations that are exponentially damped with time. On the other hand, if dephasing dominates over the Rabi oscillation, so that $\gamma_{p}>2 \Omega_{\text {eff }}$ or $\gamma_{p}=2 \Omega_{\text {eff }}, \rho_{22}$ increases monotonically. However, in all cases $\rho_{22}$ reaches a stationary value of 0.5 at long times and $\rho_{22} \sim \frac{\Omega_{\text {eff }}^{2} t^{2}}{4}$ for short times.

\section{B. Sample Computations}

The behavior of the excited state population for several values of $\gamma_{p}$ for a given value of $\Omega_{\text {eff }}$ (here chosen as $2 \pi$ ) is sketched in Fig. 11. For $\gamma_{p}<2 \Omega_{\text {eff }}$, the introduction of dephasing increases the period of the oscillation and causes the amplitudes to decay as $e^{-\frac{\gamma_{p} t}{2}}$. Although 
this is a CW laser field case, we can extract the result for the field being switched off at a specific time, i.e., a square pulsed laser which is on from $t=0$ to $t=t_{f}$, by examining the population at time $t_{f}$. (This assumes that there are no additional energy levels excited by the frequency breadth of the truncated CW source). Significantly, one can end up with an increased $\rho_{22}$ even for a larger dephasing, depending on the pulse duration. For example, assume that we turn off the field at $t=1$. If there is no dephasing, then the excited state population at $t=1$ is 0 and is thus less than that of any of the other cases with dephasing. On the other hand, for $\gamma_{p}>2 \Omega_{\text {eff }}$ (here $\gamma_{p}>4 \pi$ ), there is no oscillation; $\rho_{22}$ just increases monotonically towards 0.5 , where the system reaches the steady-state slower with increasing dephasing. If we were to consider a pulse rather than a CW laser field for this relatively strong dephasing case, the excited state population would be expected to increase up to 0.5 with the increase in the pulse duration.

Typical behavior of $\rho_{22}$ and of the 1 vs. 3 photon phase control profile (i.e., $\rho_{22}$ as a function of generic phase control variable $\Phi$ ) for several values of Rabi frequencies and $\gamma_{p}$ are shown in Fig. 2. Here we assume that the fields are abruptly turned off at the times indicated in the figure captions to produce a square pulse and the intensities are chosen so that $\left|\Omega_{h}\right|=\left|\Omega_{f}\right|$, to enhance the interference effects. The effective Rabi frequency is then $\Omega_{\mathrm{eff}}=\left|\Omega_{h}\right| \sqrt{2(1+\cos \Phi)}$. While $\Phi=0$ leads to a complete constructive interference of the two transition amplitudes, $\Phi=\pi$ leads to a complete destructive interference, i.e., no excitation from the ground to the excited state.

The typical control behavior seen in Fig. 2 depends upon the pulse duration, as well as upon $\Omega_{\mathrm{eff}}$ and $\gamma_{p}$. For example, when the field is weak and $\gamma_{p}=0$, then $\rho_{22}$ is given by [Eq (20) $-(22)]$

$$
\begin{gathered}
\rho_{22}=\frac{1}{2}\left[1-\cos \left(\Omega_{\mathrm{eff}} t\right)\right] \text { for } \gamma_{p}=0 \\
\approx \frac{1}{2}\left|\Omega_{h}\right|^{2}(1+\cos \Phi) t^{2} \quad ; \text { for } \Omega_{h} \text { small. }
\end{gathered}
$$

Hence, the system shows a "cos $\Phi$ rule". [ A similar rule obtains from Eq. (20) - Eq. (22) when $\gamma_{\mathrm{p}}<2 \Omega_{\text {eff }}$ and $s t<<1$, when $\gamma_{\mathrm{p}}>2 \Omega_{\text {eff }}$ and $\lambda_{1} t$ and $\lambda_{2} t$ are much less than one, and for $\gamma_{\mathrm{p}}=2 \Omega_{\mathrm{eff}}$ when $\gamma_{p} t / 2<<1$.] Note also that Eq. (23) predicts oscillatory behavior of $\rho_{22}$ as a function of $\Omega_{\text {eff }}$ at fixed $t$, as observed later below.

The control profiles for small $\Omega_{h}$ and $\gamma_{p}=0$ (thin dashed lines in Fig. 21) are then seen to be monotonically decreasing from the maximum excitation at $\Phi=0$, to zero excitation 
at $\Phi=\pi$, i.e. they follow the "cos $\Phi$ rule". By contrast, for strong intensity (thin solid lines in Fig. 21) in which there are many Rabi cycles during the pulse, the control curve is not necessarily monotonic since the final excited populations are determined by the time at which the fields are turned off. Introducing dephasing is seen to lead to a decreased range of control whose magnitude depends on the relative strength of the dephasing and on the effective Rabi frequencies, according to Eqs. (20) - (22).

Figure 2 demonstrates that phase control profiles are strongly dependent on the pulse duration. For weak intensities, as the pulse duration increases, the degree of control improves and the control curve continues to approximately follow a $\cos \Phi$ law (e.g., Eq. (24)). This behavior is seen both in the absence and in the presence of dephasing, although dephasing reduces the yield for a given pulse duration. In the strong field case the control profile varies strongly with pulse duration. In particular, with $\gamma_{p}=0$, if the pulse duration is smaller than the oscillation period $(=1 / 2)$ of the $\Omega_{\text {eff }}(\Phi=0)$ case, then $\rho_{22}$ decreases with increasing $\Phi$, as shown in Fig. 2(a). For the pulse duration greater than that period, the control profile no longer follows $\cos \Phi$ and the maximal yields start to appear at $\Phi \neq 0$, as shown in Figs. 2(b) to (d). In all strong intensity cases, the addition of dephasing results in a decay of $\rho_{22}$ with a rate of $e^{-\frac{\gamma_{p} t}{2}}$ for a given $\Omega_{\text {eff }}$. Thus the degree of the control worsens in the presence of dephasing as the pulse duration increases. Note that the introduction of dephasing leads to a degree of control $C$ that converges to 0.5 , where $C$ is defined as the difference between the maximum and minimum excited state populations.

Finally, we note that this treatment can be extended in two directions. The most obvious is to extend it to the general two-level $N$ photon $+M$ photon interference scenario [1] where the structure of the problem is exactly the same as that of the 1 vs. 3 photon case. The equations above therefore hold, but with the one and three photon Rabi frequencies and phases replaced by the $N$ and $M$ photon Rabi frequencies and phases. The second is to consider the more general case that includes the equal detuning of both fields from the $|1\rangle \rightarrow|2\rangle$ transition, i.e. $\delta=\omega_{21}-\omega_{h}=\omega_{21}-3 \omega_{f}$, and where the populations of levels $|2\rangle$ and $|1\rangle$ decay with the same rate $\gamma_{d}$. Then Eqs. (14) to (15) become:

$$
\begin{aligned}
& \frac{\partial \sigma_{11}}{\partial t}=-\gamma_{d}\left(\sigma_{11}-\sigma_{1 e}\right)-\operatorname{Im}\left[\Omega_{\mathrm{eff}} e^{i \theta} \sigma_{21}\right] \\
& \frac{\partial \sigma_{22}}{\partial t}=-\gamma_{d}\left(\sigma_{22}-\sigma_{2 e}\right)+\operatorname{Im}\left[\Omega_{\mathrm{eff}} e^{i \theta} \sigma_{21}\right] \\
& \frac{\partial \sigma_{21}}{\partial t}=-\left(\gamma_{d}+\gamma_{p}+i \delta\right) \sigma_{21}+\frac{i}{2}\left[\Omega_{\mathrm{eff}} e^{-i \theta}\right]\left(\sigma_{11}-\sigma_{22}\right) .
\end{aligned}
$$


Here $\sigma_{1 e}$ and $\sigma_{2 e}$ are the steady-state values of $\sigma_{11}$ and $\sigma_{22}$, respectively, when $\Omega_{\mathrm{eff}}=0$, and are introduced to allow for relaxation to equilibrium. In terms of $u, v$, and $w$, the above equations lead to

$$
\begin{array}{r}
d u / d t=-\delta v-\frac{u}{T_{2}}, \\
d v / d t=\delta u-\frac{v}{T_{2}}+\Omega_{\mathrm{eff}} w \\
d w / d t=-\frac{\left(w-w_{e}\right)}{T_{1}}+\Omega_{\mathrm{eff}} v .
\end{array}
$$

where $w_{e}=\sigma_{2 e}-\sigma_{1 e}, T_{1}=1 / \gamma_{d}$ and $T_{2}=1 /\left(\gamma_{d}+\gamma_{p}\right)$. Note that these are then of the same form as the usual Bloch equations for a monochromatic field. Torrey gave detailed analytical solutions for these equations in the monochromatic field case [28, 29] and the same analytical solutions for the 1 vs. 3 photon phase control case can be used, where the single field $\Omega$ considered by Torrey is replaced by $\Omega_{\text {eff }}$. We do not pursue this direction in this letter.

\section{SUMMARY}

In summary, we have obtained an analytic solution for $N$ vs. $M$ photon phase control of a two-level system in an environment described by a $1 / \gamma_{p}$ dephasing time, with $N=1$ and $M=3$ as a specific example. The results should serve as a prototype for understanding the results of $N$ vs. $M$ photon phase control in more complicated systems, such as controlled Xenon ionization and IBr photodissociation[30].

Acknowledgements This work was partially supported by Photonics Research Ontario and by the Natural Sciences and Engineering Research Council of Canada 
[1] P. Brumer and M. Shapiro, Principles of the Quantum Control of Molecular Processes (Wiley, New York, 2003).

[2] S. A. Rice and M. Zhao, Optical Control of Molecular Dynamics (Wiley, New York, 2000).

[3] H. Rabitz, R. de Vivie-Riedle, M. Motzkus, and K. Kompa, Science 288, 824 (2000).

[4] J. Cao, C. J. Bardeen, and K. R. Wilson, J. Chem. Phys. 113, 1898 (2000).

[5] M. Demirplak and S. A. Rice, J. Chem. Phys. 116, 8028 (2002).

[6] J. Gong and S. A. Rice, J. Chem. Phys. 120, 3777 (2004).

[7] B. D. Fainberg and V. A. Gorbunov, J. Chem. Phys. 117, 7222 (2002).

[8] M. A. Nielsen and I. L. Chuang, Quantum Computation and Quantum Information (Cambridge University Press, Cambridge, 2000).

[9] M. Shapiro and P. Brumer, Phys. Rev. A 66, 052308 (2002).

[10] P. Zanardi and M. Rasetti, Phys. Rev. Lett. 79, 3306 (1997).

[11] L. Duan and G. Guo, Phys. Rev. A 57, 737 (1998).

[12] D. A. Lidar, D. Bacon, and K. B. Whaley, Phys. Rev. Lett. 82, 4556 (1999).

[13] J. I. Cirac and P. Zoller, Phys. Rev. Lett. 74, 4091 (1995).

[14] C. J. Myatt, B. E. King, Q. A. Turchette, C. A. Sackett, D. Kielpinski, W. M. Itano, C. Monroe, and D. J. Wineland, Nature 403, 269 (2000).

[15] D. J. Tannor, R. Kosloff, and A. Bartana, Faraday Discuss. 113, 365 (1999) and references are therein.

[16] M. Shapiro, J. W. Hepburn, P. Brumer, Chem. Phys. Lett. 149, 451 (1988)

[17] C. K. Chan, P. Brumer, and M. Shapiro, J. Chem. Phys. 94, 2688 (1991).

[18] D. Petrosyan and P. Lambropoulos, Phys. Rev. Lett. 85, 1843 (2000).

[19] D. Petrosyan and P. Lambropoulos, Phys. Rev. A 63, 043417 (2001).

[20] J. C. Camparo and P. Lambropoulos, Phys. Rev. A 55, 552 (1997).

[21] C. Chen, Y-Y. Yin and D.S. Elliott, Phys. Rev. Lett. 64, 507 (1990).

[22] C. Chen, Y-Y. Yin and D.S. Elliott, Phys. Rev. Lett. 65 1737, (1990).

[23] X. Wang, R. Bersohn, K. Takahashi, M. Kawasaki, and H.L. Kim, J. Chem. Phys. 105, 2992 (1996).

[24] N.E. Karapanagioti, D. Xenakis, D. Charalambidis and C. Fotakis, J. Phys. B 29, 3599 (1996). 
[25] For a review see: R. J. Gordon, L. Zhu, and T. Seideman, Acc. Chem. Res. 32, 1007 (1999).

[26] L. K. Iwaki and D. L. Dlott, J. Phys. Chem. A. 104, 9101 (2000).

[27] H. Graener, R. Zürl and M. Hofmann, J. Phys. Chem. B. 101, 1745 (1997).

[28] H. C. Torrey, Phys. Rev. 76, 1059 (1949).

[29] L. Allen and J. H. Eberly, Optical Resonance and Two-level Atoms (Wiley, New York, 1975).

[30] H. Han and P. Brumer (manuscript in preparation). H. Han, Ph.D. Dissertation, University of Toronto, 2004. 
Figure 1. Excited state population as a function of time for various dephasing rates, $\gamma_{p}$ shown inside the box for $\Omega_{\mathrm{eff}}=2 \pi$. All the variables are in dimensionless units. Data points are connected by straight lines as a guide.

Figure 2. Excited state population versus relative phase for two different $\left|\Omega_{h}\right|$ : Solid lines and dashed lines denote the case at $\left|\Omega_{h}\right|=2 \pi$ and $\left|\Omega_{h}\right|=\pi / 5$, respectively. Thin lines and thick lines denote the case at $\gamma_{p}=0$ and $\gamma_{p}=\pi$, respectively. Fields are turned off at (a) $t=0.25$, (b) $t=0.25 \times 2$, (c) $t=0.25 \times 3$, and (d) $t=0.25 \times 8$. Note that the data in panel (d) is too widely spaced to produce the last $\rho_{22}=1$ maxima, whose exact locations can be predicted from Eq. (24). All the variables are in dimensionless units. Data points are connected by straight lines as a guide. 


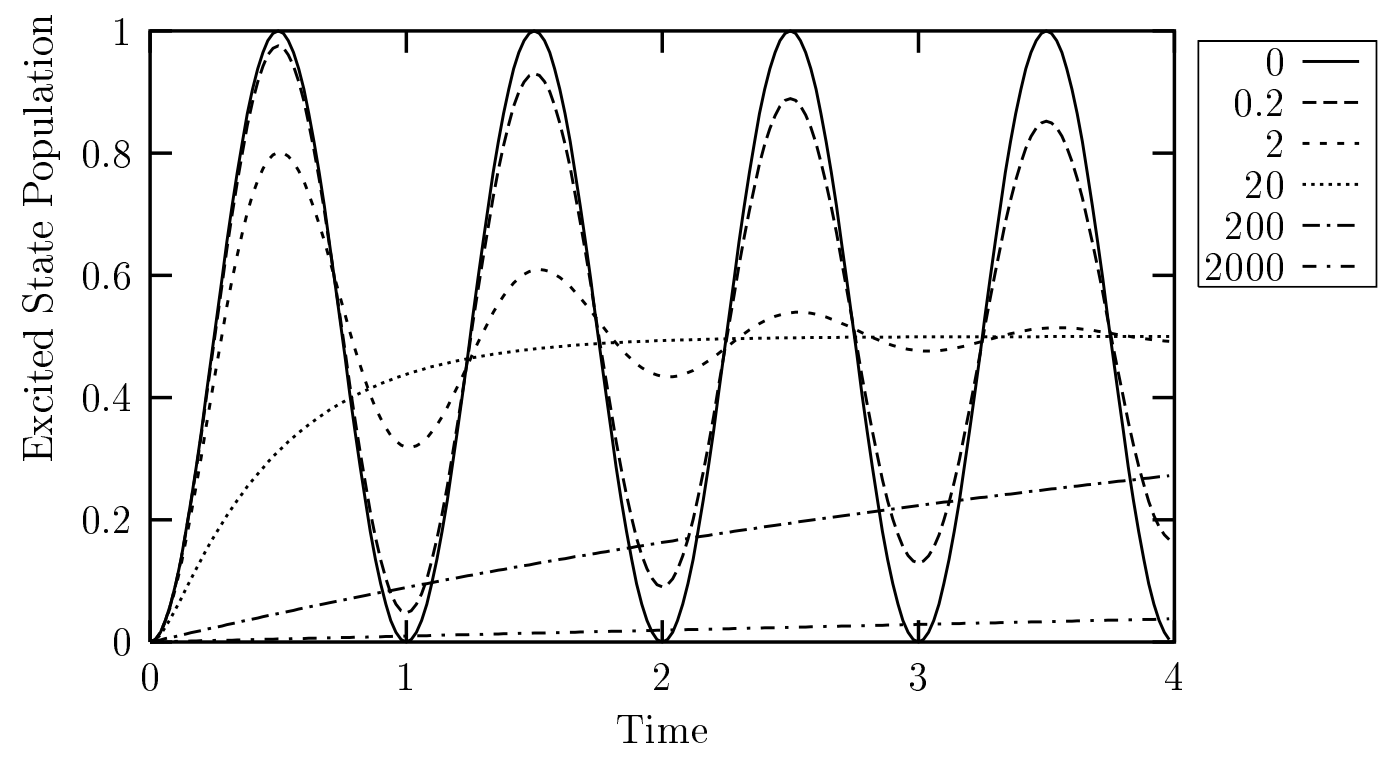

FIG. 1: 

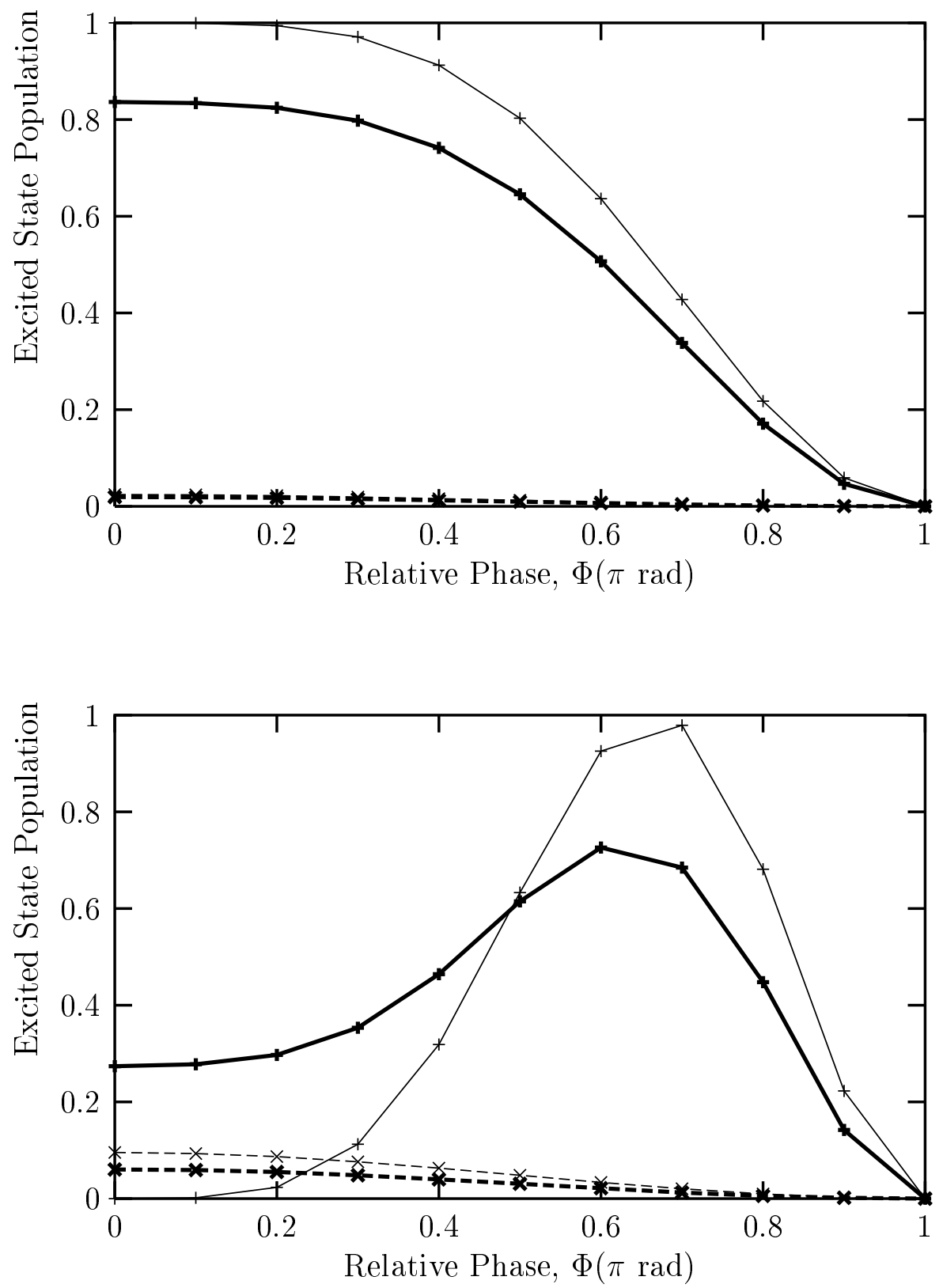

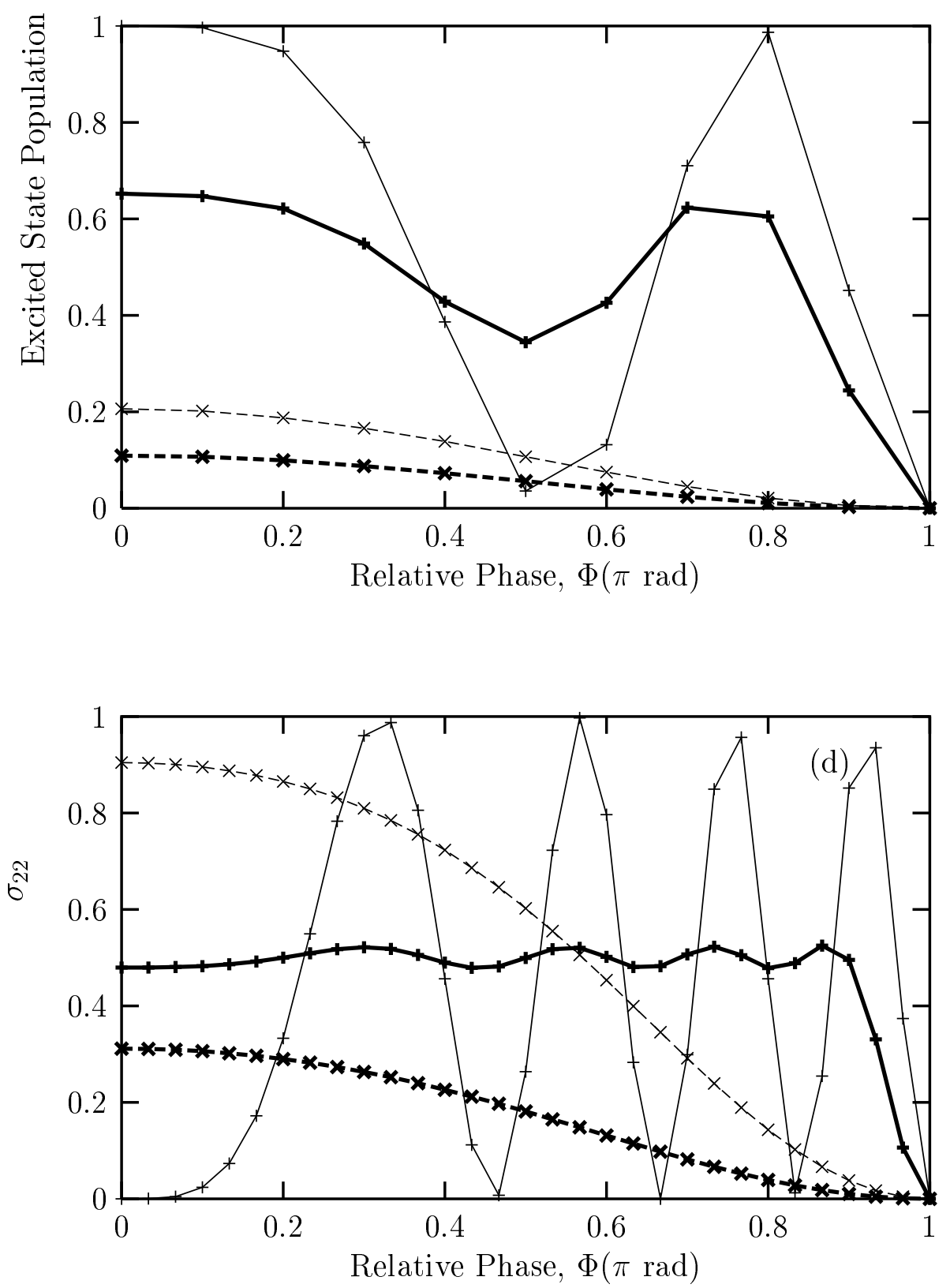

FIG. 2: 